\title{
SINGULARLY PERTURBED VOLTERRA INTEGRAL EQUATIONS WITH WEAKLY SINGULAR KERNELS
}

\author{
ANGELINA BIJURA \\ Received 9 May 2001 and in revised form 25 September 2001
}

\begin{abstract}
We consider finding asymptotic solutions of the singularly perturbed linear Volterra integral equations with weakly singular kernels. An interesting aspect of these problems is that the discontinuity of the kernel causes layer solutions to decay algebraically rather than exponentially within the initial (boundary) layer. To analyse this phenomenon, the paper demonstrates the similarity that these solutions have to a special function called the Mittag-Leffler function.
\end{abstract}

2000 Mathematics Subject Classification: 45D05, 45E10, 45A05, 33E12, 41A60.

1. Introduction. This paper considers the weakly singular scalar Volterra integral equation of the second kind

$$
\varepsilon u(t)=f(t)+\frac{1}{\Gamma(\beta)} \int_{0}^{t} \frac{k(t, s)}{(t-s)^{1-\beta}} u(s) d s, \quad 0 \leq t \leq T,
$$

where $0<\varepsilon \ll 1$ and $0<\beta<1$. The functions $f(t)$ and $k(t, s)$ are continuous and $k(t, t)=-1$. Problem (1.1) exhibits an initial layer at $t=0$ like the equations with continuous kernels, but with a narrower initial layer width of order $O\left(\varepsilon^{1 / \beta}\right)$ as $\varepsilon \rightarrow 0$.

The study on problems of type (1.1) is motivated by considering various physical problems associated with the Volterra operator including a problem on the determination of the temperature in a radiating semi-infinite solid material. For a detailed discussions on these physical problems, see Gripenberg et al. [6].

The weakly singular equation (1.1) has a solution $u(t ; \varepsilon)$ in $C[0, T]$ for all $\varepsilon>0$. For $\varepsilon=0$, (1.1) reduces to the Abel integral equation

$$
0=f(t)+\frac{1}{\Gamma(\beta)} \int_{0}^{t} \frac{k(t, s)}{(t-s)^{1-\beta}} v(s) d s, \quad 0 \leq t \leq T .
$$

It certainly does not have a continuous solution if $f(0) \neq 0$. The forcing function $f(t)$ must be smoother than the desired solution. Even if (1.2) has a solution $v(t)$ in $\mathrm{C}^{0}[0, T]$ it may not approximate $u(t ; \varepsilon)$ uniformly for $t$ in $[0, T]$ as $\varepsilon \rightarrow 0$.

Asymptotic solutions of singularly perturbed integral equations have been previously considered by Angell and Olmstead [1, 11], Lange and Smith [8, 9], and Chen [3]. But in all these, the analysis on the behaviour of formal solutions to problems with weakly singular kernels is not there. Angell and Olmstead [1] included problems with weakly singular kernels in their analysis but the way they treated this, together with 
problems of continuous kernels is not clear because as we will see, problems of type (1.1) include fractional powers in the formal series expansion.

The aim is to outline the main points underlying the processes of finding an asymptotic approximation;

$$
u(t ; \varepsilon)=y(t ; \varepsilon)+\varphi(\varepsilon) z\left(\frac{t}{\mu(\varepsilon)} ; \varepsilon\right)
$$

which are uniformly close on $[0, T]$ to $u(t ; \varepsilon)$ as $\varepsilon \rightarrow 0$. The functions $y$ and $z$ in (1.3) are respectively, the outer and inner solutions, whereas $\varphi$ and $\mu$ describe respectively, the magnitude and width respectively, of the layer region. It is stipulated that $\mu(\varepsilon) \rightarrow 0$, $\varepsilon \rightarrow 0$. The similarity that the inner solution has to the Mittag-Leffler function is the main interest of this work and is outlined carefully.

The method of additive decomposition which works for ordinary differential equations and integral equations with continuous kernel (cf. [1, 9, 12, 13, 16]) is used here. Problems of the type (1.1) do not exhibit an exponential decay in the initial layer and therefore, the methodology developed by Angell and Olmostead, and Lange and Smith, can be improved. To emphasize the fundamental ideas and illustrate the technical difficulties, we only find the leading order term $U_{0}(t ; \varepsilon)$ of the asymptotic solution. It is proved that $\left|u(t ; \varepsilon)-U_{0}(t ; \varepsilon)\right|=O(\varepsilon)$ as $\varepsilon \rightarrow 0$, on $0 \leq t \leq T$. An example from the model on thermal convection considered in [1] is solved to demonstrate the developed methodology.

In Section 2, we review some of the results which are applied later in the preceding sections. These include the hypotheses imposed on the data, solutions of Abel equations, and the Mittag-Leffler function and its asymptotic expansion.

In Section 3, we describe how the additive decomposition technique can be applied to integral equations of the type (1.1) and derive the formal solution. We only consider the leading order approximation.

In Section 4, we show that the coefficients in the formal solution derived elucidate some of their properties. We also show that if the formal solution satisfies (1.1) approximately with a residual $\rho_{0}(t ; \varepsilon)$, then $\rho_{0}(t ; \varepsilon)=O(\varepsilon)$ uniformly as $\varepsilon \rightarrow 0$, on $0 \leq t \leq T$ which implies the same condition for the formal solution $U_{0}(t ; \varepsilon)$. Finally in Section 5 , we demonstrate this methodology on the heat radiation equation (having the exact solution) modelled for a solid material with a higher thermal loss and then comment on the derivation of the first-order solution.

2. Mathematical preliminaries. The following are the hypotheses used:

(H1) $0<\beta<1$;

(H2) $k(t, s)$ is a $C^{2}$ function, if an equation like (1.1) is encountered with $k(0,0)<0$, a simple rescaling of $\varepsilon$ leads to $k(0,0)=-1$. If $k(t, t)<0$, the equation for $t \mapsto k(t, t) u(t)$ has the form of (1.1), on $\triangle_{T}$ with $k(t, t)=-1$, where

$$
\triangle_{T}=0 \leq s \leq t \leq T
$$

(H3) the function $f(t)$ is $\mathrm{C}^{2}$ on $[0, T]$ with $f(0) \neq 0$. 
2.1. Solution of Abel equations. Let $0<\beta<1$ and define the operator

$$
\left(\mathrm{D}^{\beta} \chi\right)(t):=\frac{1}{\Gamma(1-\beta)} \frac{d}{d t} \int_{0}^{t} \frac{1}{(t-s)^{\beta}} \chi(s) d s .
$$

It is a classical result of Abel's that, for $0<\beta<1$, the equation

$$
\frac{1}{\Gamma(\beta)} \int_{0}^{t} \frac{1}{(t-s)^{1-\beta}} y(s) d s=\phi(t)
$$

has the solution

$$
y(t)=\left(\mathrm{D}^{\beta} \phi\right)(t)
$$

This relies on the useful formula

$$
\int_{0}^{t} \frac{1}{(t-s)^{1-\beta} s^{\beta}} d s=\Gamma(\beta) \Gamma(1-\beta) .
$$

The following proposition follows from [10, Part II].

Proposition 2.1. If $\chi$ is continuous on $[0, T]$ and differentiable on $(0, T]$ with $\chi(0)=0$, then $\mathrm{D}^{\beta} \chi$ is also continuous on $[0, T]$ and is given by

$$
\left(\mathrm{D}^{\beta} \chi\right)(t)=\frac{1}{\Gamma(1-\beta)} \frac{d}{d t} \int_{0}^{t} \frac{1}{(t-s)^{\beta}} \chi^{\prime}(s) d s .
$$

Tonelli proved that (2.3) has a solution in $L^{1}[0, T]$ if $\phi$ is absolutely continuous on $[0, T]$. In this section, we consider the more general Abel equation

$$
\frac{1}{\Gamma(\beta)} \int_{0}^{t} \frac{k(t, s)}{(t-s)^{1-\beta}} y(s) d s=\phi(t) .
$$

Gorenflo and Vessella [5] gave several existence and uniqueness for (2.7). We state here a special case of [5, Theorem 5.1.4].

Theorem 2.2. Suppose that (H1) and (H2) hold. Let $\mathrm{D}^{\beta} \phi$ be continuous on $[0, T]$. Then (2.7) has a unique solution $y$ in $\mathrm{C}[0, T]$ and

$$
\|y\|_{C^{1}} \leq C\left\|\mathrm{D}^{\beta} \phi\right\|
$$

for some constant $C>0$ depending on $T$ and $\|k\|_{C^{1}\left(\Delta_{T}\right)}$.

Later, we will require the knowledge of the asymptotic behaviour of solutions $y(t)$ of (2.7) for small $t$. The following result is [5, Theorem 5.1.5] and comes from [2].

THEOREM 2.3. Suppose that (H1) and (H2) hold. Suppose that there is a function $\tilde{\phi}(t)$ in $C^{1}$ such that $\phi(t)=t^{\mu} \tilde{\phi}(t)$, with $1-\beta+\mu>0$. Then (2.7) has a unique solution $y(t)$, and this solution can be expressed as

$$
y(t)=t^{\mu-\beta} \tilde{y}(t)
$$


where $\tilde{y}(t)=v+t y^{*}(t)$ with $v$ constant and $y^{*}$ continuous. Moreover, $v=0$ if and only if $\tilde{\phi}(0)=0$, and there is a constant $c>0$ such that

$$
\|\tilde{y}\|_{C} \leq c\|\tilde{\phi}\|_{C^{1}}
$$

2.2. The Mittag-Leffler function and its asymptotic expansion. We present here some of the properties of the Mittag-Leffler function, $E_{\mu}: \mathbb{C} \rightarrow \mathbb{C}$. In particular, we state formulae for $E_{\mu}(z)$ for large $z \in \mathbb{C}$. For each $\mu>0$, the Mittag-Leffler function is defined by

$$
E_{\mu}(z)=\sum_{n=0}^{\infty} \frac{1}{\Gamma(\mu n+1)} z^{n}
$$

$E_{\mu}$ is entire, and

$$
E_{1}(z)=e^{z}, \quad E_{2}(z)=\cosh z, \quad E_{1 / 2}\left(z^{1 / 2}\right)=2 \pi^{-1 / 2} e^{-z} \operatorname{erfc}\left(-z^{1 / 2}\right) .
$$

An interesting property proved by Pollard [15] is that, $t \mapsto E_{\mu}(-t)$ is completely monotonic on $[0, \infty)$ if $0 \leq \mu \leq 1$. Thus for $\mu$ in this parameter range, $(-1)^{n} E_{\mu}^{(n)}(-t) \geq 0$ for $t \geq 0$, where

$$
E_{\mu}^{n}(z)=\frac{d^{n} E_{\mu}}{d z^{n}}(z)
$$

A detailed discussion on the properties of the Mittag-Leffler function can be found in Erdélyi et al. [4, Chapter 18] or Paris and Kaminski [14, Chapter 5].

We are interested in the asymptotic expansion of $E_{\mu}(z)$ only in case where $0<$ $\mu<1$. However, the asymptotic expansions formulae below are for all $0<\mu<2$. These expansions are derived from the representation

$$
E_{\mu}(z)=\frac{1}{2 \pi i} \int_{c-i \infty}^{c+i \infty} e(s) z^{-s} d s
$$

for some $0<c<1$ where

$$
e(s)=\frac{\pi \cos \pi s}{\Gamma(1-\mu s) \sin \pi s},
$$

(2.14) is derived from the formula for inverting a Mellin transform. We decompose the path in (2.14) into a contour $C^{\prime}$ which is closed to the left. It is shown in Figure 2.1. Now $e(s) z^{-s}$ has simple poles at $s=0,-1,-2, \ldots$ Let $a_{n}$ be the residue of $s \mapsto e(s) z^{-s}$ at $-n$. Then

$$
a_{n}=\frac{z^{n}}{\Gamma(1+\mu n)} .
$$

To check that $e(s)$ above is the proper choice in (2.14),

$$
\frac{1}{2 \pi i} \int_{C^{\prime}} e(s) z^{-s} d s=\sum_{n=0}^{\infty} a_{n}=\sum_{n=0}^{\infty} \frac{z^{n}}{\Gamma(\mu n+1)}=E_{\mu}(z) .
$$




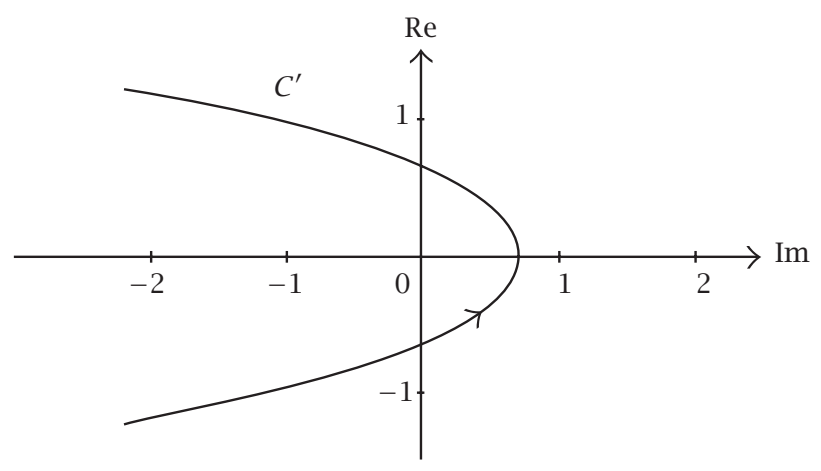

FIGURE 2.1. The contour of integration for the Mittag-Leffler function $E_{\mu}(z)$.

Using the integral representation in (2.14), it is shown in $[4,14]$ that for $0<\mu<2$, the controlling factor of the leading behaviour of $E_{\mu}(z)$ is $e^{z^{1 / \mu}}$ as $z \rightarrow \infty$. Stokes lines occur at $\operatorname{Re} z^{1 / \mu}=0$ or $\arg z= \pm \pi \mu$ and anti-Stokes lines occur at $\operatorname{Im} z^{1 / \mu}=0$ or $\arg z= \pm(\pi / 2) \mu$.

It is shown in $[4,14]$ that the expansion of $E_{\mu}(z)$ when $\mu<2$ is given by

$$
\begin{aligned}
& E_{\mu}(z) \sim \frac{1}{\mu} e^{z^{1 / \mu}}-\sum_{k=1}^{\infty} \frac{z^{-k}}{\Gamma(1-\mu k)}, \quad|\arg z|<\frac{3 \pi \mu}{2}, \\
& E_{\mu}(z) \sim-\sum_{k=1}^{\infty} \frac{z^{-k}}{\Gamma(1-\mu k)}, \quad|\arg (-z)|<\frac{\pi}{2}(2-\mu) .
\end{aligned}
$$

It should be noted that (2.18) is a valid asymptotic expansion in the Poincaré sense. The discussion in [14, Section 5.1] elucidates what is happening for $0<\mu<1$. The expansions have a common sectors $\pi \mu / 2<|\arg z|<3 \pi \mu / 2$. In the sector $|\arg z|<\pi \mu$, expansion (2.18a) is valid. However, the exponential term is decaying for $\pi \mu / 2<$ $|\arg z|<\pi \mu$ since the anti-Stokes lines at $\arg z= \pm \pi \mu / 2$ have been crossed. $E_{\mu}(z)$ is exponentially large as $|z| \rightarrow \infty$ for $|\arg z|<\pi \mu / 2$. As $\arg z$ crosses the Stokes lines $\arg z= \pm \pi \mu$, the exponential term disappears from the leading order term and becomes subdominant. It reemerges as $\arg z$ crosses $\pm 2 \pi \mu$, but it is exponentially decaying. At $\arg z=3 \pi \mu / 2$, expansion (2.18a) is no longer valid. Expansion (2.18b) holds for $|\arg (-z)|<\pi \mu / 2$. Since we are interested in the asymptotic expansion on the negative real axis, this sector particularly concerns us. The conclusion is that we obtain the composite expansion

$$
\begin{aligned}
& E_{\mu}(z) \sim \frac{1}{\mu} e^{z^{1 / \mu}}-\sum_{k=1}^{\infty} \frac{z^{-k}}{\Gamma(1-\mu k)}, \quad|\arg z|<\pi \mu, \\
& E_{\mu}(z) \sim-\sum_{k=1}^{\infty} \frac{z^{-k}}{\Gamma(1-\mu k)}, \quad|\arg (-z)|<\pi(1-\mu) .
\end{aligned}
$$

We illustrate this in Figures 2.2 and 2.3. 
2.3. Solution of a simple class of Abel-Volterra equations. The Abel-Volterra equation

$$
z(\tau)=\psi(\tau)+\frac{v}{\Gamma(\beta)} \int_{0}^{\tau} \frac{1}{(\tau-\sigma)^{1-\beta}} z(\sigma) d \sigma, \quad \tau \geq 0,
$$

has an explicit solution in terms of the Mittag-Leffler function $E_{\beta}$.

The following existence and uniqueness result, which is attributed to Hille and Tamarkin [7], is given in Geronflo and Vessella [5].

THEOREM 2.4. Let $0<\beta<1, v$ any real number, and let $\psi(\tau)$ be continuous on $[0, \infty)$. Then (2.20) has the continuous solution $z(\tau)$ given by

$$
z(\tau)=\frac{d}{d \tau} \int_{0}^{\tau} E_{\beta}\left(v(\tau-\sigma)^{\beta}\right) \psi(\sigma) d \sigma, \quad \tau \geq 0 .
$$

The function $z$ is unique in the class $\mathrm{L}_{\mathrm{loc}}^{\infty}\left(\mathbb{R}^{+}\right)$.

3. Heuristic analysis and formal solution. The analysis of the additive decomposition shows that we should introduce the new time scale $\tau=t / \mu(\varepsilon)$, we call this the inner variable and seek an asymptotic solution $u(t ; \varepsilon)$ in the form of (1.3). The function $\varphi(\varepsilon)$ describes the magnitude of the initial layer, whereas $\mu(\varepsilon)$ describes the width. Using the dominant balancing technique, it is easily found that if $f(0) \neq 0$ then the magnitude of the boundary layer is $\varepsilon^{-1}$ and the width is $\varepsilon^{\gamma}$. We, therefore, seek an asymptotic solution $u(t ; \varepsilon)$ in the form

$$
u(t ; \varepsilon)=y(t ; \varepsilon)+\frac{1}{\varepsilon} z\left(\frac{t}{\varepsilon^{\gamma}} ; \varepsilon\right)
$$

and require that

$$
\lim _{\tau \rightarrow \infty} z(\tau ; \varepsilon)=0
$$

The function $z\left(t / \varepsilon^{\gamma} ; \varepsilon\right)$ corrects the nonuniformity in the initial layer. Substituting (3.1) into (1.1) gives

$$
\begin{aligned}
\varepsilon y(t ; \varepsilon)+z\left(\frac{t}{\varepsilon^{\gamma}} ; \varepsilon\right)= & f(t)+\frac{1}{\Gamma(\beta)} \int_{0}^{t} \frac{k(t, s)}{(t-s)^{1-\beta}} y(s ; \varepsilon) d s \\
& +\frac{1}{\Gamma(\beta) \varepsilon} \int_{0}^{t} \frac{k(t, s)}{(t-s)^{1-\beta}} z\left(\frac{s}{\varepsilon^{\gamma}} ; \varepsilon\right) d s .
\end{aligned}
$$

It is assumed that $y(t ; \varepsilon)$ and $z(\tau ; \varepsilon)$ have asymptotic expansions of the form

$$
y(t ; \varepsilon) \sim \sum_{n=0}^{\infty} \varepsilon^{n} y_{n}(t), \quad z(\tau ; \varepsilon) \sim \sum_{n=0}^{\infty} \varepsilon^{n y} z_{n}(\tau),
$$

as $\varepsilon \rightarrow 0$, so that

$$
u(t ; \varepsilon) \sim \sum_{n=0}^{\infty} \varepsilon^{n} y_{n}(t)+\sum_{n=0}^{\infty} \varepsilon^{n \gamma-1} z_{n}\left(\frac{t}{\varepsilon^{\gamma}}\right) .
$$




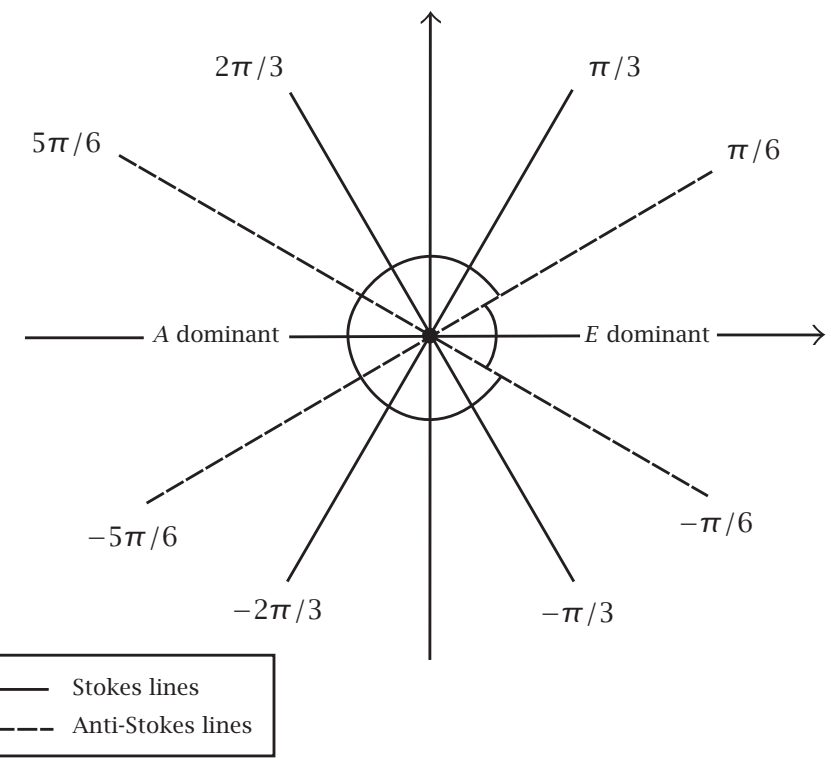

FIGURE 2.2. The Stokes lines are shown for the exponential term in (2.19a) corresponding to $\mu=1 / 3$. Also shown is the sector $E$ where the exponential term in (2.19a) dominates and the sector $A$ where the algebraic term in (2.19) dominates.

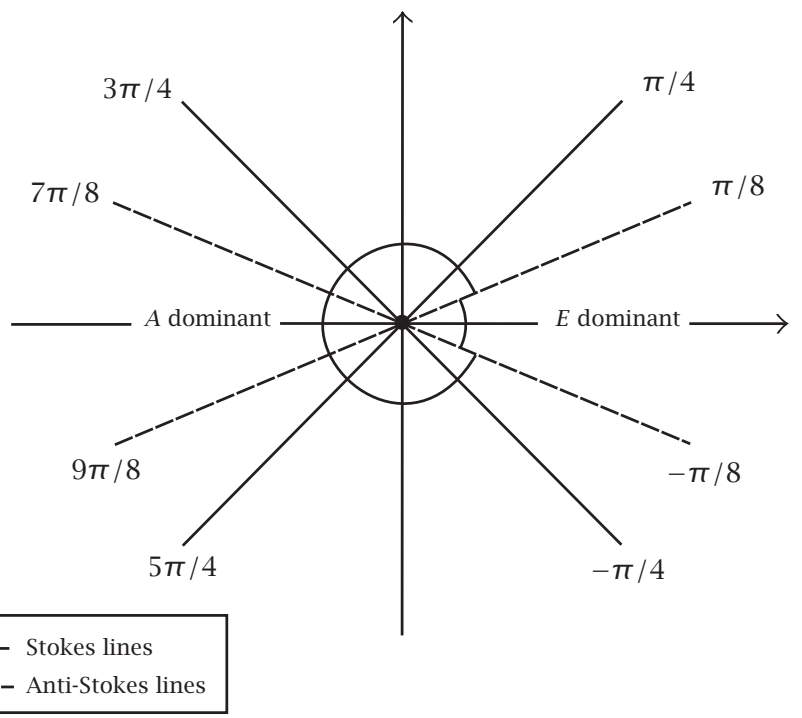

FIGURE 2.3. The Stokes lines are shown for the exponential term in (2.19a) corresponding to $\mu=1 / 4$. Also shown is the sector $E$ where the exponential term in (2.19a) dominates and the sector $A$ where the algebraic term in (2.19) dominates. 
Moreover, we require that for all $n \geq 0$,

$$
\lim _{\tau \rightarrow \infty} z_{n}(\tau)=0
$$

Firstly, we restrict attention to

$$
U_{0}(t ; \varepsilon)=y_{0}(t)+\frac{1}{\varepsilon} z_{0}\left(\frac{t}{\varepsilon^{\gamma}}\right)
$$

assuming that

$$
z_{0}(\tau) \longrightarrow \frac{f(0)}{\Gamma(1-\beta) \tau^{\beta}} \quad \text { as } \tau \longrightarrow \infty
$$

Suppose that $U_{0}(t ; \varepsilon)$ satisfies (1.1) approximately, with a residual $\rho_{0}(t ; \varepsilon)$, then

$$
\begin{aligned}
\rho_{0}(t ; \varepsilon)+\varepsilon y_{0}(t)+z_{0}\left(\frac{t}{\varepsilon^{\gamma}}\right)= & f(t)+\frac{1}{\Gamma(\beta)} \int_{0}^{t} \frac{k(t, s)}{(t-s)^{1-\beta}} y_{0}(s) d s \\
& +\frac{1}{\varepsilon \Gamma(\beta)} \int_{0}^{t} \frac{k(t, s)}{(t-s)^{1-\beta}} z_{0}\left(\frac{s}{\varepsilon^{\gamma}}\right) d s .
\end{aligned}
$$

By expressing this in terms of $\tau=t / \varepsilon^{\gamma}$,

$$
\begin{aligned}
\rho_{0}\left(\varepsilon^{\gamma} \tau ; \varepsilon\right)+z_{0}(\tau)+\varepsilon y_{0}\left(\varepsilon^{\gamma} \tau\right)= & f\left(\varepsilon^{\gamma} \boldsymbol{\tau}\right)+\frac{1}{\Gamma(\beta)} \int_{0}^{\tau} \frac{k\left(\varepsilon^{\gamma} \boldsymbol{T}, \varepsilon^{\gamma} \sigma\right)}{(\tau-\sigma)^{1-\beta}} z_{0}(\sigma) d \sigma \\
& +\varepsilon \int_{0}^{\tau} \frac{k\left(\varepsilon^{\gamma} \tau, \varepsilon^{\gamma} \sigma\right)}{(\tau-\sigma)^{1-\beta}} y_{0}\left(\varepsilon^{\gamma} \sigma\right) d \sigma .
\end{aligned}
$$

This can be rearranged as

$$
\begin{aligned}
\rho_{0}\left(\varepsilon^{\gamma} \tau ; \varepsilon\right)+\varepsilon y_{0}\left(\varepsilon^{\gamma} \tau\right)= & \left(f(0)+\frac{1}{\Gamma(\beta)} \int_{0}^{\tau} \frac{k(0,0)}{(\tau-\sigma)^{1-\beta}} z_{0}(\sigma) d \sigma-z_{0}(\tau)\right) \\
& +f\left(\varepsilon^{\gamma} \boldsymbol{T}\right)-f(0)+\frac{1}{\Gamma(\beta)} \int_{0}^{\tau} \frac{k\left(\varepsilon^{\gamma} \tau, \varepsilon^{\gamma} \sigma\right)-k(0,0)}{(\tau-\sigma)^{1-\beta}} z_{0}(\sigma) d \sigma \\
& +\frac{\varepsilon}{\Gamma(\beta)} \int_{0}^{\tau} \frac{k\left(\varepsilon^{\gamma} \tau, \varepsilon^{\gamma} \sigma\right)}{(\tau-\sigma)^{1-\beta}} y_{0}\left(\varepsilon^{\gamma} \sigma\right) d \sigma,
\end{aligned}
$$

and hence

$$
\rho_{0}\left(\varepsilon^{\gamma} \tau ; \varepsilon\right)=\left(f(0)+\frac{1}{\Gamma(\beta)} \int_{0}^{\tau} \frac{k(0,0)}{(\tau-\sigma)^{1-\beta}} z_{0}(\sigma) d \sigma-z_{0}(\tau)\right)+O(\varepsilon)+O\left(\varepsilon^{\gamma}\right) .
$$

We see that if $\rho_{0}\left(\varepsilon^{\gamma} \tau ; \varepsilon\right)=o(1)$ as $\varepsilon \rightarrow 0$ for fixed $\tau>0$, then

$$
z_{0}(\tau)=f(0)-\frac{1}{\Gamma(\beta)} \int_{0}^{\tau} \frac{1}{(\tau-\sigma)^{1-\beta}} z_{0}(\sigma) d \sigma, \quad \tau \geq 0 .
$$

To derive the leading order outer solution, we express (3.13) in terms of $t=\varepsilon^{\gamma} \tau$ and substitute into (3.9), giving

$$
\begin{aligned}
\rho_{0}(t ; \varepsilon)+\varepsilon y_{0}(t)= & f(t)-f(0)+\frac{1}{\Gamma(\beta)} \int_{0}^{t} \frac{k(t, s)}{(t-s)^{1-\beta}} y_{0}(s) d s \\
& +\frac{1}{\varepsilon \Gamma(\beta)} \int_{0}^{t} \frac{k(t, s)-k(0,0)}{(t-s)^{1-\beta}} z_{0}\left(\frac{s}{\varepsilon^{\gamma}}\right) d s .
\end{aligned}
$$


It follows from (3.8) and the dominated convergence theorem that

$$
\frac{1}{\varepsilon} \int_{0}^{t} \frac{\{k(t, s)-k(0,0)\}}{(t-s)^{1-\beta}} z_{0}\left(\frac{s}{\varepsilon^{\gamma}}\right) d s \rightarrow \frac{f(0)}{\Gamma(1-\beta)} \int_{0}^{t} \frac{\{k(t, s)-k(0,0)\}}{(t-s)^{1-\beta} s^{\beta}} d s,
$$

as $\varepsilon \rightarrow 0$. If $\rho(t ; \varepsilon)=o(1)$ as $\varepsilon \rightarrow 0$, we deduce from (3.9) that the leading order outer solution $y_{0}(t)$ satisfies

$$
0=f(t)-f(0)+\frac{f(0)}{\Gamma(\beta) \Gamma(1-\beta)} \int_{0}^{t} \frac{k(t, s)-k(0,0)}{(t-s)^{1-\beta} s^{\beta}} d s+\frac{1}{\Gamma(\beta)} \int_{0}^{t} \frac{k(t, s)}{(t-s)^{1-\beta}} y_{0}(s) d s .
$$

If $y_{0}(t)$ satisfies (3.16) and $z_{0}(\tau)$ obeys (3.13), it follows from (3.14) that

$$
\rho_{0}(t ; \varepsilon)=-\varepsilon y_{0}(t)+\frac{1}{\varepsilon \Gamma(\beta)} \int_{0}^{t} \frac{k(t, s)-k(0,0)}{(t-s)^{1-\beta}}\left(z_{0}\left(\frac{s}{\varepsilon^{\gamma}}\right)-\frac{f(0) \varepsilon}{\Gamma(1-\beta) s^{\beta}}\right) d s .
$$

4. Properties of the formal solution. We show here that solutions of equations for $y_{0}(t)$ and $z_{0}(\tau)$ exist and satisfy the assumed properties.

Equation (3.16) for the outer solution can be rewritten as

$$
0=\phi(t)+\int_{0}^{t} \frac{k(t, s)}{(t-s)^{1-\beta}} y_{0}(s) d s, \quad 0 \leq t \leq T
$$

where

$$
\phi(t)=f(t)-f(0)+\frac{f(0)}{\Gamma(\beta) \Gamma(1-\beta)} \int_{0}^{t} \frac{k(t, s)-k(0,0)}{(t-s)^{1-\beta} s^{\beta}} d s
$$

Note that

$$
\left|\frac{f(0)}{\Gamma(\beta) \Gamma(1-\beta)} \int_{0}^{t} \frac{\{k(t, s)-k(0,0)\}}{(t-s)^{1-\beta} s^{\beta}} d s\right| \leq f(0) \sup _{0 \leq s \leq t}|k(t, s)-k(0,0)| \longrightarrow 0
$$

as $t \rightarrow 0$. This and (H3) imply that $\phi(0)=0$. Also

$$
\begin{aligned}
\frac{1}{t} \int_{0}^{t} \frac{k(t, s)-k(0,0)}{(t-s)^{1-\beta} s^{\beta}} d s & =\frac{1}{t} \int_{0}^{1} \frac{\{k(t, t \theta)-k(0,0)\}}{(1-\theta)^{1-\beta} \theta^{\beta}} d \theta \\
& \longrightarrow \partial_{1} k(0,0) \int_{0}^{1} \frac{1}{(1-\theta)^{1-\beta} \theta^{\beta}} d \theta+\partial_{2} k(0,0) \int_{0}^{1} \frac{\theta^{(1-\beta)}}{(1-\theta)^{1-\beta}} d \theta
\end{aligned}
$$

as $t \rightarrow 0$, where $\partial_{1}$ and $\partial_{2}$ are the operators of partial differentiation. Hence, we can write

$$
\phi(t)=t \tilde{\phi}(t)
$$

and show that $\tilde{\phi}(t)$ is $C^{1}$. Using Theorem 2.3, we can establish from (4.1) and (4.5) the following proposition. 
Proposition 4.1. Suppose that (H1), (H2), and (H3) hold. Then (3.16) has a unique continuous solution $y_{0}(t)$ which satisfies

$$
y_{0}(t)=t^{1-\beta} \tilde{y}_{0}(t)
$$

where $\tilde{y}_{0}$ is continuous on $[0, T]$.

It is a simple corollary of Theorem 2.4 and (2.19b) that the following result is true.

Proposition 4.2. Suppose that (H1), (H2), and (H3) hold. Then (3.13) has the continuous solution

$$
z_{0}(\tau)=f(0) E_{\beta}\left(-\tau^{\beta}\right)
$$

for $\tau \geq 0$, which satisfies

$$
z_{0}(\tau) \sim f(0) \sum_{j=1}^{\infty}(-1)^{j+1} \frac{\tau^{-\beta j}}{\Gamma(1-\beta j)} \quad \text { as } \tau \longrightarrow \infty .
$$

REMARK 4.3. This results vindicates assumption (3.8) made in the derivation of (3.16) and (3.13) or $y_{0}(t)$ and $z_{0}(\tau)$.

Next, we prove that if $\rho_{0}(t ; \varepsilon)$ satisfies (3.17), then there exists a positive constant $\tilde{C}$ independent of $\varepsilon$ such that

$$
\left|\left(\mathrm{D}^{\beta} \rho_{0}\right)(t ; \varepsilon)\right| \leq \tilde{C} \varepsilon, \quad \varepsilon \longrightarrow 0,
$$

on $0 \leq t \leq T$.

If we apply the operator $\mathrm{D}^{\beta}$ into (3.17), we get

$$
\begin{aligned}
\left(\mathrm{D}^{\beta} \rho_{0}\right)(x ; \varepsilon)= & -\varepsilon\left(\mathrm{D}^{\beta} y_{0}\right)(x) \\
& +\frac{1}{\varepsilon \Gamma(\beta) \Gamma(1-\beta)} \frac{d}{d x} \int_{0}^{x}(x-t)^{-\beta} \int_{0}^{t} \frac{k(t, s)-k(0,0)}{(t-s)^{1-\beta}} z_{0}\left(\frac{s}{\varepsilon^{\gamma}}\right) d s d t \\
& -\frac{f(0)}{\Gamma(\beta) \Gamma(1-\beta)^{2}} \frac{d}{d x} \int_{0}^{x}(x-t)^{-\beta} \int_{0}^{t} \frac{k(t, s)-k(0,0)}{(t-s)^{1-\beta} s^{\beta}} d s d t .
\end{aligned}
$$

A similar argument used in (3.15) implies that when $\varepsilon$ is sufficiently small,

$$
\left|\left(\mathrm{D}^{\beta} \rho_{0}\right)(t ; \varepsilon)\right| \leq \varepsilon\left|\left(\mathrm{D}^{\beta} y_{0}\right)(t)\right|, \quad 0 \leq t \leq T .
$$

Propositions 2.1 and 4.1 imply that $\left(\mathrm{D}^{\beta} y_{0}\right)(t)$ is continuous and, therefore, there exists a constant $\tilde{C}$ such that (4.9) is satisfied.

Let $R_{0}(t ; \varepsilon)=u(t ; \varepsilon)-U_{0}(t ; \varepsilon)$. Then $R_{0}(t ; \varepsilon)$ satisfies

$$
\varepsilon R_{0}(t ; \varepsilon)=\rho_{0}(t ; \varepsilon)+\frac{1}{\Gamma(\beta)} \int_{0}^{t} \frac{k(t, s)}{(t-s)^{1-\beta}} R_{0}(s ; \varepsilon) d s .
$$

The existence theorems on Volterra integral equations guarantee the existence of a unique continuous function, $R_{0}(t ; \varepsilon), \varepsilon>0$ on $[0, T]$ which solves this equation. 
To prove that $\left|R_{0}(t ; \varepsilon)\right|=O(\varepsilon)$, as $\varepsilon \rightarrow 0$, let $h(t, s)=k(t, s)-k(0,0)$, then the above equation is equivalent to

$$
\frac{1}{\Gamma(\beta)} \int_{0}^{t} \frac{R_{0}(s ; \varepsilon)}{(t-s)^{1-\beta}} d s=-\varepsilon R_{0}(t ; \varepsilon)+\rho_{0}(t ; \varepsilon)+\frac{1}{\Gamma(\beta)} \int_{0}^{t} \frac{h(t, s)}{(t-s)^{1-\beta}} R_{0}(s ; \varepsilon) d s .
$$

Applying the operator $\mathrm{D}^{\beta}$ to (4.13), we get

$$
R_{0}(x ; \varepsilon)=-\varepsilon\left(\mathrm{D}^{\beta} R_{0}\right)(x ; \varepsilon)+\left(\mathrm{D}^{\beta} \rho_{0}\right)(x ; \varepsilon)+\int_{0}^{x} L_{h}(x, s) R_{0}(s ; \varepsilon) d s,
$$

where

$$
L_{h}(x, s)=\frac{1}{\Gamma(\beta) \Gamma(1-\beta)} \int_{s}^{x} \frac{\partial}{\partial t}\left(\frac{h(t, s)}{(t-s)^{1-\beta}}\right) \frac{d t}{(x-t)^{\beta}}
$$

is continuous as long as $h$ is continuous. It then follows that

$$
\left|R_{0}(t ; \varepsilon)\right|=\varepsilon\left|\left(\mathrm{D}^{\beta} R_{0}\right)(t ; \varepsilon)\right|+\left|\left(\mathrm{D}^{\beta} \rho_{0}\right)(t ; \varepsilon)\right|+\int_{0}^{t}\left|L_{h}(t, s)\right|\left|R_{0}(s ; \varepsilon)\right| d s .
$$

Since $R_{0}(0 ; \varepsilon)=\rho_{0}(0 ; \varepsilon)=0$, Proposition 2.1 implies that $\mathrm{D}^{\beta} R_{0}$ is continuous on $[0, T]$ and

$$
\varepsilon\left|\left(\mathrm{D}^{\beta} R_{0}\right)(t ; \varepsilon)\right| \leq\left|\left(\mathrm{D}^{\beta} \rho_{0}\right)(t ; \varepsilon)\right|+\int_{0}^{t}\left|L_{k}(t, s)\right|\left|R_{0}(s ; \varepsilon)\right| d s .
$$

Substituting (4.17) into (4.16) yields

$$
\left|R_{0}(t ; \varepsilon)\right| \leq 2\left|\left(\mathrm{D}^{\beta} \rho_{0}\right)(t ; \varepsilon)\right|+\int_{0}^{t}\left\{\left|L_{h}(t, s)\right|+\left|L_{k}(t, s)\right|\right\}\left|R_{0}(s ; \varepsilon)\right| d s .
$$

Then the usual Gronwall's inequality and (4.9) imply that there exists a constant $C_{0}>0$ which does not depend on $\varepsilon$ such that

$$
\left|R_{0}(t ; \varepsilon)\right| \leq \varepsilon C_{0}, \quad \varepsilon \longrightarrow 0
$$

on $[0, T]$.

5. A demonstrated example. Angell and Olmstead in [1] consider the following weakly singular linear singularly perturbed Volterra equation which follows from thermal convection:

$$
\varepsilon u(t)=f(t)-\frac{1}{\pi^{1 / 2}} \int_{0}^{t} \frac{u(s)}{(t-s)^{1 / 2}} d s,
$$

where

$$
f(t)=\frac{1}{\pi^{1 / 2}} \int_{0}^{t} \frac{h(s)}{(t-s)^{1 / 2}} d s,
$$

and $h(t)$ is $C^{2}$ with $h(0) \neq 0$. Since $\Gamma(1 / 2)=\pi^{1 / 2}$, this corresponds to (1.1) with $k(t, s)=-1$ and $\beta=1 / 2$. Therefore $\gamma=2$. The exact solution of (5.1) can be obtained by Laplace transforms or read directly from (2.12) and (2.21). It is given by

$$
u(t ; \varepsilon)=\frac{f(t)}{\varepsilon}-\frac{1}{\varepsilon^{2}} \int_{0}^{t} e^{(t-s) / \varepsilon^{2}} \operatorname{erfc}\left(\frac{(t-s)^{1 / 2}}{\varepsilon}\right) h(s) d s .
$$


Since $f\left(\varepsilon^{2} \tau\right)=2 \varepsilon h(0) \tau^{1 / 2} / \pi^{1 / 2}+O\left(\varepsilon^{2}\right)$, we look for an asymptotic solution of the form

$$
u(t ; \varepsilon)=\sum_{j=0}^{\infty}\left(\varepsilon^{j} y_{j}(t)+\varepsilon^{2 j} z_{j}\left(\frac{t}{\varepsilon^{2}}\right)\right) .
$$

Following the formal method of Section 3, it is found that the leading order outer solution $y_{0}(t)$ obeys

$$
0=\int_{0}^{t} \frac{h(s)-y_{0}(s)}{(t-s)^{1 / 2}} d s, \quad t \geq 0
$$

and hence $y_{0}(t)=h(t)$.

The inner correction term $z_{0}(\tau)$ is a solution of

$$
\begin{aligned}
z_{0}(\tau) & =-y_{0}(0)+\frac{2 \tau^{1 / 2}}{\pi^{1 / 2}}\left(h(0)-y_{0}(0)\right)-\frac{1}{\pi^{1 / 2}} \int_{0}^{\tau} \frac{1}{(\tau-\sigma)^{1 / 2}} z_{0}(\sigma) d \sigma \\
& =-h(0)-\frac{1}{\pi^{1 / 2}} \int_{0}^{\tau} \frac{1}{(\tau-\sigma)^{1 / 2}} z_{0}(\sigma) d \sigma .
\end{aligned}
$$

By (2.12) and (2.21),

$$
z_{0}(\tau)=-h(0) e^{\tau} \operatorname{erfc}\left(\tau^{1 / 2}\right), \quad \tau \geq 0
$$

The asymptotic expansion of the integral

$$
\operatorname{erfc} \sqrt{\boldsymbol{\tau}}=\frac{2}{\sqrt{\pi}} \int_{\sqrt{\tau}}^{\infty} e^{-t^{2}} d t \sim \frac{e^{-\tau}}{\sqrt{\pi \tau}}\left\{1-\frac{1}{2 \tau}+\frac{3}{4 \tau^{2}}+\cdots\right\} \quad \text { as } \tau \rightarrow \infty
$$

implies that

$$
z_{0}(\tau) \sim-\frac{h(0)}{\sqrt{\pi \tau}}\left\{1-\frac{1}{2 \tau}+\frac{3}{4 \tau^{2}}+\cdots\right\}
$$

so that $z_{0}(\tau) \rightarrow 0$ as $\tau \rightarrow \infty$, but only algebraically.

Therefore, up to the leading order, the formal solution of (5.1) is given by

$$
U_{0}(t ; \varepsilon)=h(t)-h(0) e^{t / \varepsilon^{2}} \operatorname{erfc}\left(\frac{t}{\varepsilon^{2}}\right)
$$

To show directly that $U_{0}(t ; \varepsilon)$ approximates the solution of (5.3) to within $O(\varepsilon)$, consider the difference

$$
\begin{aligned}
u(t ; \varepsilon)-U_{0}(t ; \varepsilon)= & \frac{1}{\varepsilon} \int_{0}^{t} \frac{h(s)}{\pi^{1 / 2}(t-s)^{1 / 2}} d s \\
& -\frac{1}{\varepsilon^{2}} \int_{0}^{t} e^{(t-s) / \varepsilon^{2}} \operatorname{erfc}\left(\frac{(t-s)^{1 / 2}}{\varepsilon}\right) h(s) d s \\
& -h(t)+h(0) e^{t / \varepsilon^{2}} \operatorname{erfc}\left(\frac{t^{1 / 2}}{\varepsilon}\right)
\end{aligned}
$$


Integrating by parts

$$
\begin{aligned}
\frac{1}{\varepsilon^{2}} \int_{0}^{t} e^{(t-s) / \varepsilon^{2}} \operatorname{erfc}\left(\frac{\sqrt{t-s}}{\varepsilon}\right) h(s) d s \\
=h(0) e^{t / \varepsilon^{2}} \operatorname{erfc} \frac{\sqrt{t}}{\varepsilon}-h(t)+\frac{1}{\varepsilon} \int_{0}^{t} \frac{h(s)}{\sqrt{\pi(t-s)}} d s+\varepsilon^{2} h^{\prime}(0) e^{t / \varepsilon^{2}} \operatorname{erfc} \frac{\sqrt{t}}{\varepsilon}-\varepsilon^{2} h^{\prime}(t) \\
-\varepsilon \int_{0}^{t}[\pi(t-s)]^{-1 / 2} h^{\prime}(s) d s \\
-\varepsilon^{2} \int_{0}^{t}\left\{e^{(t-s) / \varepsilon^{2}} \operatorname{erfc} \frac{\sqrt{t-s}}{\varepsilon}-\frac{2 \varepsilon \sqrt{t-s}}{\sqrt{\pi}}\right\} h^{\prime \prime}(s) d s
\end{aligned}
$$

Substituting this into (5.11), we get

$$
\begin{aligned}
u(t ; \varepsilon)-U_{0}(t ; \varepsilon)= & -\varepsilon \int_{0}^{t}[\pi(t-s)]^{-1 / 2} h^{\prime}(s) d s-\varepsilon^{2} h^{\prime}(t)+\varepsilon^{2} e^{t / \varepsilon^{2}} \operatorname{erfc} \frac{\sqrt{t}}{\varepsilon} h^{\prime}(0) \\
& -\varepsilon^{2} \int_{0}^{t}\left\{e^{(t-s) / \varepsilon^{2}} \operatorname{erfc} \frac{\sqrt{(t-s)}}{\varepsilon}-\frac{2 \varepsilon \sqrt{(t-s)}}{\sqrt{\pi}}\right\} h^{\prime \prime}(s) d s .
\end{aligned}
$$

This implies that

$$
\left|u(t ; \varepsilon)-U_{0}(t ; \varepsilon)\right|=O(\varepsilon)
$$

as $\varepsilon \rightarrow 0$ uniformly on $0 \leq t \leq T$.

We now examine the exact solution (5.3) with the view of directly determining a valid asymptotic solution for $u(t ; \varepsilon)$. Suppose now that $h(t)$ is $\mathrm{C}^{\infty}$. For the outer expansion, we fix $t>0$ in (5.3) and let $\varepsilon \rightarrow 0$. Then

$$
u(t ; \varepsilon) \sim \sum_{n=0}^{\infty} \varepsilon^{n} v_{n}(t) \quad \text { as } \varepsilon \longrightarrow 0 .
$$

The integration by parts in (5.12) gives

$$
v_{0}(t)=h(t), \quad v_{1}(t)=\frac{h(0)}{(\pi t)^{1 / 2}}-\int_{0}^{t} \frac{1}{\pi^{1 / 2}(t-s)^{1 / 2}} h^{\prime}(s) d s, \ldots,
$$

where the first term in $v_{1}$ follows from the first term in (5.12) and the asymptotic expansion (5.8). To get the inner expansion, we express (5.3) in terms of the inner variable $\tau=t / \varepsilon^{2}$ to get

$$
u\left(\varepsilon^{2} \tau ; \varepsilon\right):=w(\tau ; \varepsilon)=\int_{0}^{\tau}\left\{\frac{1}{\pi^{1 / 2}(\tau-\sigma)^{1 / 2}}-e^{\tau-\sigma} \operatorname{erfc}(\tau-\sigma)^{1 / 2}\right\} h\left(\varepsilon^{2} \sigma\right) d \sigma .
$$

This suggests that the inner expansion has the form

$$
w(\tau ; \varepsilon)=\sum_{n=0}^{\infty} \varepsilon^{2 n} w_{n}(\tau) \quad \text { as } \varepsilon \longrightarrow 0
$$


Equating the coefficients of like powers of $\varepsilon$, we get

$$
w_{n}(\tau)=\frac{h^{(n)}(0)}{n !} \int_{0}^{\tau}\left\{\frac{1}{\pi^{1 / 2}(\tau-\sigma)^{1 / 2}}-e^{\tau-\sigma} \operatorname{erfc}(\tau-\sigma)^{1 / 2}\right\} \sigma^{n} d \sigma .
$$

The leading order term in (5.18) is given by

$$
w_{0}(\tau)=h(0) \int_{0}^{\tau}\left\{\frac{1}{\pi^{1 / 2}(\tau-\sigma)^{1 / 2}}-e^{\tau-\sigma} \operatorname{erfc}(\tau-\sigma)^{1 / 2}\right\} d \sigma,
$$

equivalently,

$$
w_{0}(\tau)=h(0)-h(0) e^{\tau} \operatorname{erfc} \sqrt{\tau}
$$

The first-order term $w_{1}(\tau)$ is given by

$$
w_{1}(\tau)=h^{\prime}(0) \int_{0}^{\tau}\left\{\frac{1}{\pi^{1 / 2}(\tau-\sigma)^{1 / 2}}-e^{\tau-\sigma} \operatorname{erfc}(\tau-\sigma)^{1 / 2}\right\} \sigma d \sigma,
$$

which on integration by parts is

$$
w_{1}(\tau)=h^{\prime}(0) \tau-h^{\prime}(0) \int_{0}^{\tau} e^{\sigma} \operatorname{erfc} \sqrt{\sigma} d \sigma
$$

We then see that the leading order term in the outer expansion and the leading order term in the inner expansion form a composite expansion which is the uniformly valid asymptotic solution $U_{0}(t ; \varepsilon)$ obtained by the methodology developed.

REMARK 5.1. It is important to establish the asymptotic behaviour of $y_{0}(t)$ as $t \downarrow 0$ and $z_{0}(\tau)$ as $\tau \rightarrow \infty$. If we define $w(\tau ; \varepsilon):=\varepsilon u\left(\varepsilon^{\gamma} \tau ; \varepsilon\right)$, then

$$
w(\tau ; \varepsilon)=f\left(\varepsilon^{\gamma} \tau\right)+\int_{0}^{\tau} \frac{k\left(\varepsilon^{\gamma} \tau, \varepsilon^{\gamma} \sigma\right)}{(\tau-\sigma)^{1-\beta}} w(\sigma ; \varepsilon) d \sigma .
$$

Therefore, we expect the inner expansion to be

$$
w(\tau ; \varepsilon) \sim \sum_{j=0}^{\infty} \varepsilon^{j \gamma} w_{j}(\tau) \quad \text { as } \varepsilon \longrightarrow 0 .
$$

Comparing this to (3.5) we see that

$$
w_{0}(\tau)+\varepsilon^{\gamma} w_{1}(\tau)+\cdots \sim z_{0}(\tau)+\varepsilon y_{0}\left(\varepsilon^{\gamma} \tau\right)+\cdots .
$$

Since (4.6) implies that $\varepsilon y_{0}\left(\varepsilon^{\gamma} \tau\right)=\varepsilon^{\gamma} T^{1-\beta} \tilde{y}_{0}\left(\varepsilon^{\gamma} \tau\right)$, the apparent anomaly of a $O(\varepsilon)$ term balancing with an $O\left(\varepsilon^{\gamma}\right)$ term does not arise. In fact the equation for $z_{1}(\tau)$ can be found to be

$$
z_{1}(\tau)=\psi_{1}(\tau)+\frac{1}{\Gamma(\beta)} \int_{0}^{\tau} \frac{k(0,0)}{(\tau-\sigma)^{1-\beta}} z_{1}(\sigma) d \sigma,
$$

where $\psi_{1}$ is continuous. The required asymptotic expansion for large $\tau$ is established with the assumption that $\psi_{1}(\tau)$ assumes a power series expansion in $\tau$. 
However, for the outer solution, the abnormality of $O(\varepsilon)$ term balancing with $O\left(\varepsilon^{\gamma}\right)$ term is there. Thus the equation for $y_{1}(t)$ depends on the actual value of $\gamma$.

ACKNOWLEDGments. The author is grateful to Dr. David Reynolds for his accurate suggestions and to Prof. Igor Barashenkov for his encouragement.

\section{REFERENCES}

[1] J. S. Angell and W. E. Olmstead, Singularly perturbed Volterra integral equations, SIAM J. Appl. Math. 47 (1987), no. 1, 1-14.

[2] K. E. Atkinson, An existence theorem for Abel integral equations, SIAM J. Math. Anal. 5 (1974), 729-736.

[3] M. Chen, Singular perturbation analysis of nonlinear Fredholm integral equation with weak singularity, Z. Angew. Math. Phys. 23 (1972), 266-271.

[4] A. Erdélyi, W. Magnus, F. Oberhettinger, and F. G. Tricomi, Higher Transcendental Functions. Vol. III, McGraw-Hill, New York, 1955.

[5] R. Gorenflo and S. Vessella, Abel Integral Equations, Lecture Notes in Mathematics, vol. 1461, Springer-Verlag, Berlin, 1991.

[6] G. Gripenberg, S.-O. Londen, and O. Staffans, Volterra Integral and Functional Equations, Encyclopedia of Mathematics and Its Applications, Cambridge University Press, Cambridge, 1990.

[7] E. Hille and J. D. Tamarkin, On the theory of linear integral equations, Ann. of Math. 31 (1930), 479-528.

[8] C. G. Lange and D. R. Smith, Singular perturbation analysis of integral equations, Stud. Appl. Math. 79 (1988), no. 1, 1-63.

[9] _ Singular perturbation analysis of integral equations. II, Stud. Appl. Math. 90 (1993), no. 1, 1-74.

[10] P. Linz, Analytical and Numerical Methods for Volterra Equations, SIAM Studies in Applied Mathematics, vol. 7, SIAM, Pennsylvania, 1985.

[11] W. E. Olmstead and J. S. Angell, Singularly perturbed integral equations with endpoint boundary layers, SIAM J. Appl. Math. 49 (1989), no. 6, 1567-1584.

[12] R. E. O'Malley Jr., Introduction to Singular Perturbations, Applied Mathematics and Mechanics, vol. 14, Academic Press, New York, 1974.

[13] _ Singular Perturbation Methods for Ordinary Differential Equations, Applied Mathematical Sciences, vol. 89, Springer-Verlag, New York, 1991.

[14] R. B. Paris and D. Kaminski, Asymptotics and Mellin-Barnes Integrals, Encyclopedia of Mathematics and Its Applications, vol. 85, Cambridge University Press, Cambridge, 2001.

[15] H. Pollard, The completely monotonic character of the Mittag-Leffler function $E_{a}(-x)$, Bull. Amer. Math. Soc. 54 (1948), 1115-1116.

[16] D. R. Smith, Singular-Perturbation Theory. An Introduction with Applications, Cambridge University Press, Cambridge, 1985.

ANGELINA BiJURA: DEPARTMENT OF MATHEMATICS, UNIVERSITY OF DAR ES SALAAM, P.O. BOX 35062, DAR ES SALAAM, TANZANIA

Current address: DEPARTMENT OF MATHEMATICS AND APPLIED MATHEMATICS, UNIVERSITY of CAPE TOWN, RONDEBOSCH 7701, SOUTH AFRICA

E-mail address: abijura@cs.udsm.ac.tz, abijura@maths.uct.ac.za 


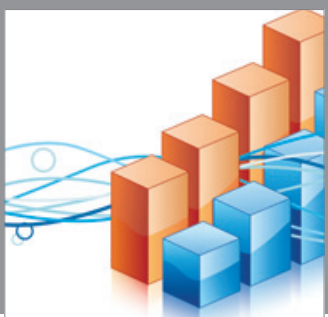

Advances in

Operations Research

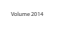

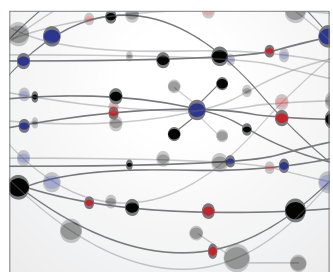

\section{The Scientific} World Journal
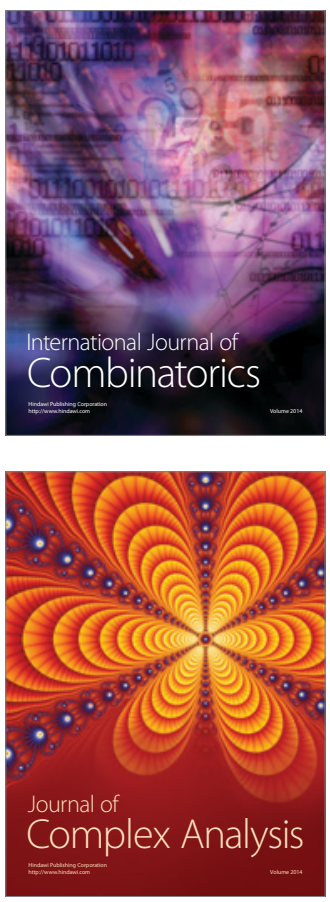

International Journal of

Mathematics and

Mathematical

Sciences
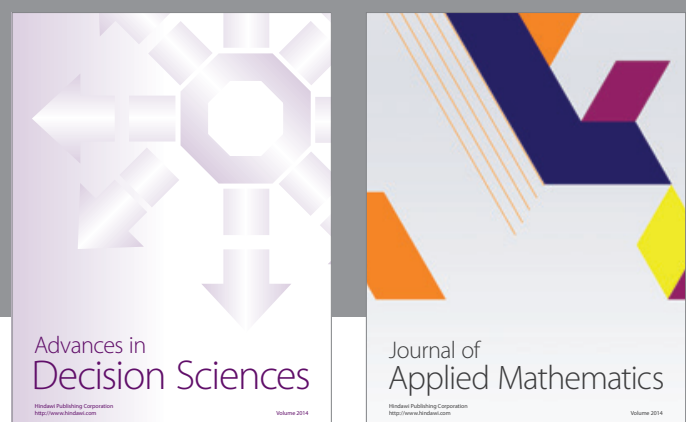

Journal of

Applied Mathematics
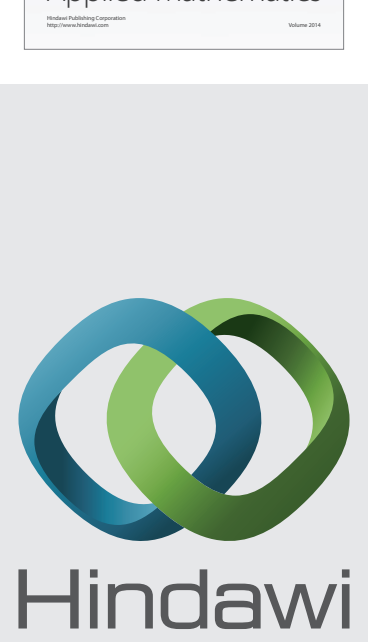

Submit your manuscripts at http://www.hindawi.com
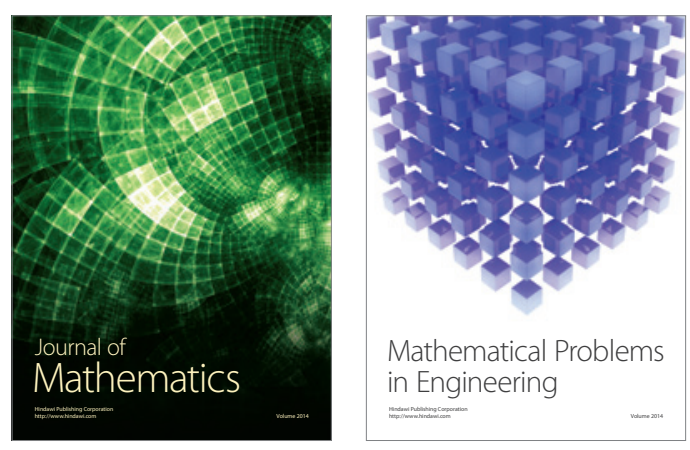

Mathematical Problems in Engineering
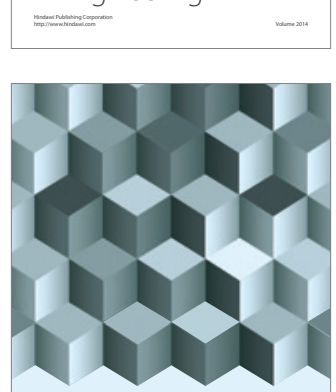

Journal of

Function Spaces
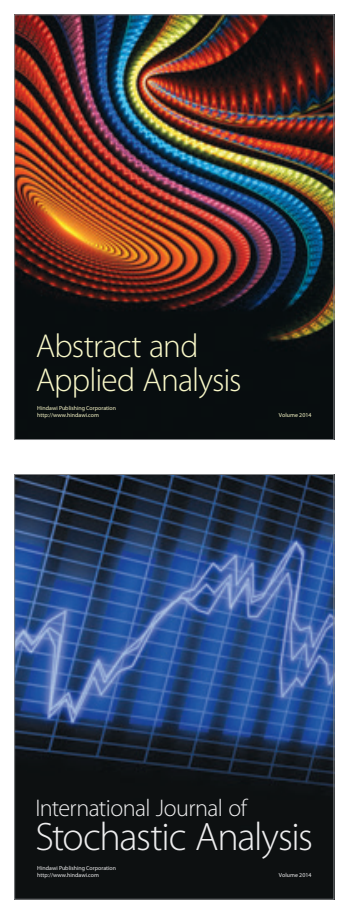

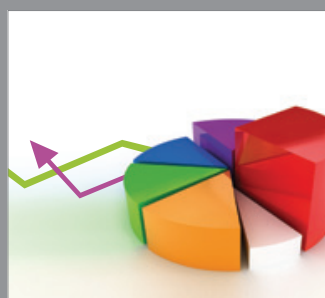

ournal of

Probability and Statistics

Promensencen
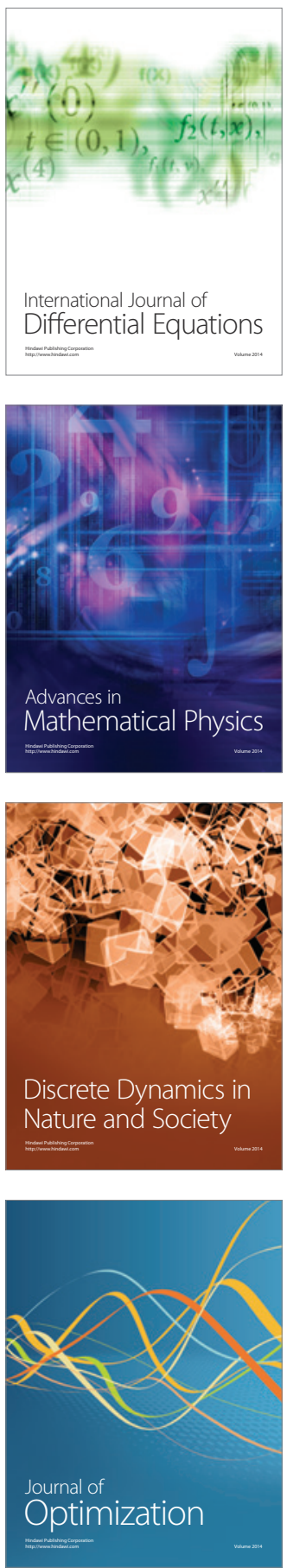